Produto \& Produção, vol 17 n 2, p 53-70, 2016

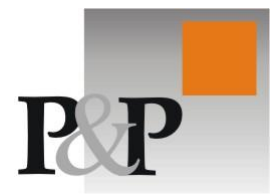

RECEBIDO EM 26/11/2014. ACEITO EM 01/06/2016.

\author{
Patrícia Belfiore Fávero \\ Universidade Federal do $A B C$ - UFABC \\ pbfavero@usp.br
}

Fabio Augusto Mollik Zoucas

Centro Universitário da Fundação Educacional Inaciana "Padre Sabóia de Medeiros" Centro Universitário da FEI

fabiozoucas@hotmail.com

\title{
Redes neurais para previsão da produção industrial de diferentes segmentos
}

\begin{abstract}
RESUMO
Este trabalho tem como objetivo propor um modelo de rede neural para previsão de séries de produção de onze segmentos industriais brasileiros. Primeiramente, estudou-se diferentes tipos de redes que vêm sendo implementadas na literatura nos últimos anos. Estudando o comportamento dessas séries de produção e as principais características de cada tipo de rede, concluímos que a rede Perceptron MultiCamadas com atraso no tempo (TDNN) é a melhor para o cálculo e análise da previsão da produção dos onze segmentos escolhidos do setor industrial. A rede neural foi então aplicada considerando duas diferentes estratégias de modelo estrutural. Concluímos que o modelo de rede neural proposto foi eficaz na previsão de séries de produção de segmentos industriais.
\end{abstract}

Palavras-chave: Redes neurais; Previsão de Séries Temporais; Indústria.

\begin{abstract}
This paper aims to propose a neural network model for forecasting the production time series of eleven different industries in Brazil. Firstly, we study different types of networks that have been implemented in the literature in recent years. Studying the behavior of these time series and the main characteristics of the each network type, we conclude that the Multi-Layer Perceptron network with delay in time (TDNN) is the best to estimate the production time series of eleven industrial segments. The neural network was then applied considering two different strategies of structural model. We conclude that the neural network model proposed was effective for forecasting production time series in these industries.
\end{abstract}

Keywords: Neural networks; Forecasting; Industry. 


\section{Introdução}

A previsão de vendas tem um papel importante na estratégia de negócios. Uma previsão de vendas eficaz pode ajudar as empresas a reduzir custos de produção e determinar o preço de venda do produto (Kuo e Xue, 1998; Armstrong, 2006). Para Slack et al. (2009), um sistema eficaz de previsão é essencial para o planejamento da capacidade, mas é também crucial para compreender a incerteza da demanda, pois permite prever os riscos do nível de serviço.

De acordo com Werner e Ribeiro (2003), o estudo de previsões de séries de tempo é uma importante atividade, pois revela tendências de mercado, contribui para o planejamento estratégico, ajuda a resolver problemas de forma mais imediata, permite um melhor entendimento do comportamento dos clientes e constitui fonte de informação adicional nas decisões de suporte de investimentos e tamanho da equipe.

Para Stevenson (2001), o melhor sistema de previsão não é necessariamente o mais acurado, e também não o mais barato; reflete, na verdade, a melhor combinação de acurácia e custo.

Características do sistema de previsão, especialmente nos setores industriais, tem um comportamento flexível e são dependentes de numerosas variáveis, sendo, muitas vezes, inerente ao processo (Byrne et al., 2011). Os métodos tradicionais são aplicados para dados com comportamento sazonal, cíclico e com tendência, não sendo adequados para dados aleatórios. Desta forma, os modelos de redes neurais têm sido comumente utilizados para prever o comportamento de um conjunto de dados que não pode ser tratado pelas técnicas convencionais.

Redes Neurais Artificiais (RNA) são estruturas flexíveis que têm sido aplicadas para uma grande variedade de problemas de previsão com alto grau de acuracidade, sendo sua principal vantagem a habilidade de modelar sistemas não lineares. Entretanto, um modelo de rede neural necessita uma grande quantidade de dados históricos até alcançar um alto nível de acuracidade (Khashei et al., 2008; Crone et al., 2011).

Redes neurais também podem ser definidas como um sistema computacional contendo muitas unidades não lineares simples ou nós interconectados por links. A previsão de séries de tempo usando Redes Neurais Artificiais tem sido muito atrativa, uma vez que seus caminhos são representados internamente pela rede e o paralelismo natural inerente à sua arquitetura cria a possibilidade de melhoria da performance comparado com modelos tradicionais (Braga et al., 2007).

De acordo com o mesmo autor, a habilidade da rede neural de aprender a partir de seu ambiente através de um processo iterativo de ajuste de seus pesos, o treinamento, e então dar respostas coerentes em relação aos dados desconhecidos, é uma demonstração que sua aplicação vai além do mapeamento dos dados de entrada e análise dos dados de saída.

O objetivo deste trabalho é propor um modelo de rede neural e verificar a aplicação efetiva desta ferramenta para prever as séries de produção de onze segmentos industriais. A construção das redes e os modelos existentes para diferentes atividades serão detalhados neste trabalho.

\section{Redes Neurais Artificiais}

De acordo com Braga et. al. (2007), as Redes Neurais Artificiais são sistemas paralelos distribuídos compostos por unidades de processamento simples (neurônios artificiais) que calculam determinadas funções matemáticas (normalmente não lineares) e dispostas em uma ou mais camadas interligadas por um grande número de conexões, geralmente unidirecionais e que possuem duas fases de processamento, a de aprendizado e a da utilização.

Numa rede neural, os dados entram na primeira camada e os resultados processados são declarados no último nó da camada de saída, sendo descoberta pela modificação dos pesos atribuídos a ela.

Segundo Wray et. al. (1994), a análise de redes neurais tem três vantagens primárias. A primeira é que o desenvolvimento da rede não necessita do conhecimento das suas relações fundamentais entre as variáveis de entrada e saída. A segunda é que a sua habilidade associativa a torna mais robusta e em condições de trabalhar com situações de perdas ou incertezas dos dados. 
Adicionalmente, o desempenho das redes neurais não é prejudicado por problemas de multicolinearidade.

A combinação de neurodinâmica e arquitetura definem o paradigma da rede neural, e na teoria, uma rede com uma camada escondida e um número suficiente de neurônios escondidos atende a qualquer função contínua (KAASTRA e BOYD, 1996).

Conforme Aguiar et al. (2007), a classificação fundamental das estruturas neurais pode ser feita em relação ao método de propagação da informação recebida, sendo que a distinção está no tipo de alimentação apresentada (propagação para frente ou feedforward e realimentação ou recurrent), conforme figura 1 .

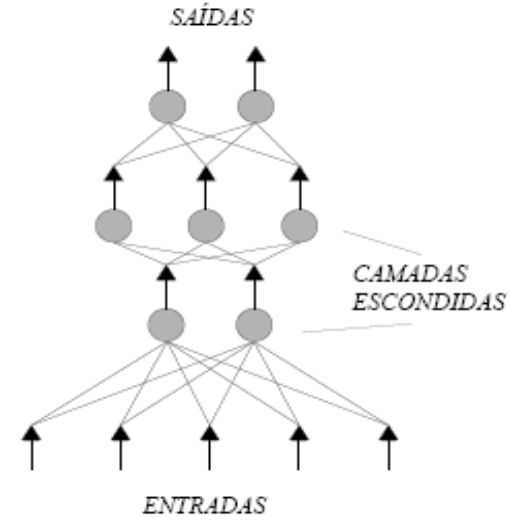

Propagação para Frente

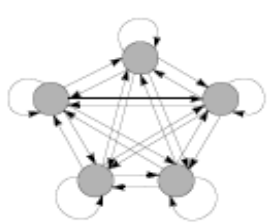

Realimentação

Figura 1 - Tipo de Alimentação de Redes Neurais

A propriedade importante das redes neurais é a habilidade de aprender e de melhorar seu desempenho através do ajuste de seus parâmetros, chamado de treinamento da rede. Esse treinamento pode ser classificado como supervisionado ou não supervisionado (Aguiar et al., 2007).

Segundo os autores, os algoritmos de aprendizado são um conjunto de regras bem definidas para a solução de um problema e para as redes neurais, todo conhecimento está armazenado nas sinapses, ou seja, nos pesos atribuídos às conexões entre os neurônios.

Para Braga et al. (2007), a função de ativação de uma rede neural é a responsável por gerar a saída resultante da aplicação dos vetores de peso pela entrada da rede. A figura 2 mostra exemplos de funções de ativação mais comuns.

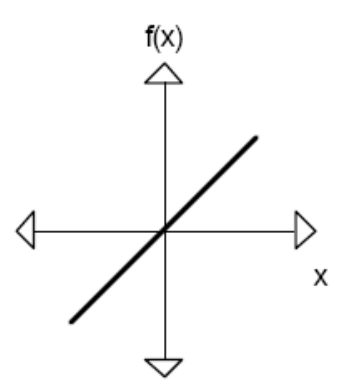

FUNÇÃO LINEAR
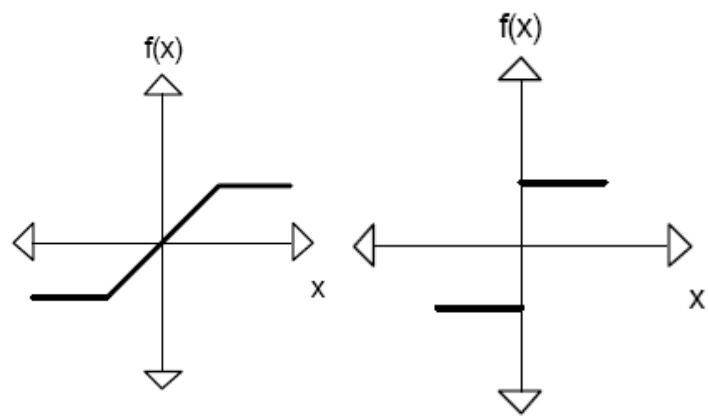

FUNÇÃO RAMPA
FUNÇÃO DEGRAU

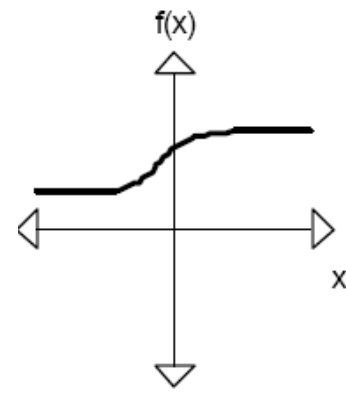

FUNÇÃO SIGMOIDAL

Figura 2 - Exemplos de Função de Ativação 
Existem diversos modelos de redes neurais desenvolvidos a partir do modelo original proposto por McCulloch e Pitts (1943), tais como o Perceptron, o Adaline (Adaptative Linear Network), o Perceptron de Multicamadas (Feedforward), as redes BAM (Memória Bi-Direcional Associativa) e ART (Adaptative Ressonance Theory), rede probabilística, o modelo de Hopfield, o modelo de Kohonem, e a rede TDNN (Time Delay Neural Network) que foi aplicada no estudo e será detalhada neste artigo.

Os diferentes modelos de redes neurais se diferenciam pela sua arquitetura, o modelo de algoritmo, a quantidade de camadas e a sua função de transferência (Kourentzes, 2013; Cao et al., 2012).

Descreveremos a seguir os principais conceitos das redes mencionadas acima com base em Braga et al. (2007) e Aguiar et al. (2007).

O Perceptron foi desenvolvido por Rosenblatt e é caracterizado por uma rede com uma única camada cujos pesos e erros podem ser treinados para se obter um vetor esperado, dado um vetor de entrada.

Nas redes lineares, também chamado de modelo Adaline (Adaptative Linear Network), os neurônios têm função de transferência linear, possibilitando que a saída seja um número real e não apenas 0 ou 1. Ela é inicializada com pesos aleatórios e a rede calcula os pesos de acordo com os dados de entrada e saída. A solução é obtida pelo valor do somatório dos erros ao quadrado e a regra de treinamento, é feita pelo cálculo das mudanças dos pesos de uma camada linear que façam decrescer a soma dos quadrados dos erros da camada. Calcula-se repetidamente o erro entre as entradas e saídas até que a soma do quadrado dos erros atinja um valor aceitável.

No caso de redes Perceptron com múltiplas camadas não é possível obter o erro diretamente através da diferença entre a saída desejada e a saída recorrente da rede, pois não existem saídas desejadas definidas para camadas intermediárias. O problema é calcular ou estimar o erro das camadas intermediárias. Qualquer Perceptron com pelo menos uma camada escondida é um Perceptron Multicamada. Cada neurônio recebe várias entradas da camada anterior e calcula uma combinação linear dessas variáveis.

As Redes BAM (Memória Bi-Direcional Associativa) são redes hetero-associativa e de conteúdo endereçável, consistindo de duas camadas. Ela usa o fluxo de informação forward e backward para produzir uma pesquisa associativa em resposta a um estímulo.

As redes ART (Adaptive Ressonance Theory) são redes de arquitetura que aprendem em tempo real os códigos de representação estável em resposta a uma sequência arbitrária de padrões de entrada. Neste tipo de rede, o aprendizado é não supervisionado e é tratado como uma ação dinâmica e incremental, com objetivo de alcançar novos padrões na entrada.

A rede probabilística é usada para classificação, e quando uma entrada é apresentada, a primeira camada calcula as distâncias entre os vetores de entrada e os esperados e obtém um vetor cujos elementos indicam a distância entre a entrada e o exemplar usado para treinamento. A segunda camada soma essas contribuições para cada classe de entradas e obtém um vetor de probabilidades. Uma função de transferência na saída da camada 2 exibe o máximo dessas probabilidades e assume 1 para essa classe e 0 para as demais.

Já o modelo de Hopfield é uma rede com realimentação, onde os valores de entrada são realimentados pelo resultado da rede através de um comportamento dinâmico.

O Modelo de Kohonem é uma rede neural não supervisionada que usa neurônios adaptativos para receber sinais de um evento espacial, consistindo em medidas ou dados, como frequência ou situação. Tal modelo tem os neurônios da camada de saída disputando entre si a representação da informação apresentada aos neurônios de entrada.

Redes Neurais com Atraso no Tempo ou TDNN (Time Delay Neural Networks) são do tipo múltiplas camadas cujos neurônios das camadas intermediárias e de saída são replicados ao longo do tempo. As redes TDNN recebem este nome porque os dados da série temporal passam por atrasos de tempo, de forma que nas entradas dos neurônios seja apresentada uma sequência de dados correspondente a uma janela de tempo fixo, que se desloca ao longo da série, conforme a Figura 18.

\section{Metodologia}


Para demonstrar a efetiva aplicação de redes neurais no cálculo de previsão das séries de produção, foram analisadas as séries de tempo de dados reais de 11 segmentos industriais de janeiro de 1980 a setembro de 2013. Os dados foram extraídos do site do IBGE (Instituto Brasileiro de Geografia e Estatística).

Os dados de cada série de tempo referem-se a um tipo de categoria de produção conforme seu segmento, sendo eles: Farmacêutica, Extrativa Mineral, Indústria de Transformação, Indústria Geral, Indústria Têxtil, Bens de Capital, Intermediários, Consumo, Consumo Duráveis, Consumo Não Duráveis e Indústria de Bebidas. As séries históricas podem ser consultadas diretamente no SIDRA (Sistema IBGE de Recuperação Automática) ou no próprio site do IBGE.

Através da função de auto-correlação de cada segmento, define-se a melhor estrutura de rede compatível com um modelo genérico apresentado, e assim, utilizando um algoritmo de uso comercial (compatível com os dados utilizados), aplica-se a rede neural para o cálculo da previsão de demanda.

Foi feita uma análise preliminar das séries temporais de modo a verificar sua estacionariedade e sazonalidade. A partir do cálculo da estimativa da função auto-correlação para todas as séries temporais dos diversos segmentos, verificou-se que todas as séries históricas consideradas neste trabalho são séries temporais não-estacionárias.

Desta forma, escolheu-se as redes neurais TDNN (Time Delay Neural Network), formada por Percepton Multicamadas, capazes de trabalhar com séries não-estacionárias e até mesmo com comportamentos sazonais. Outra estratégia utilizada para eliminar o efeito da sazonalidade foi aplicar a mesma topologia de redes neurais para dados transformados, tornando-os séries temporais estacionárias, que consiste na rede RNN (Recurrent Neural Network). Assim, tanto os dados de entrada como o de saída são diferenças entre dois meses, separados por um intervalo de 12 meses, ou seja, diferenças entre mesmo mês em anos consecutivos.

O algoritmo utilizado para o treinamento foi o Levenberg-Marquardt, que é bastante eficiente para este tipo de aplicação. Para a modelagem da rede neural, bem como o desenvolvimento do algoritmo de simulação, foi utilizado o software Matlab.

Para cada um dos segmentos apresentados, os resultados da saída da rede serão analisados e comparados com a saída desejada (dados reais conhecidos) por meio de medidas de erro, justificando assim a eficácia da aplicação da técnica de Redes Neurais no cálculo de previsão de séries de tempo do segmento industrial.

\section{Modelo Proposto}

As séries históricas apresentadas correspondem a um período de 405 meses para cada um dos onze segmentos estudados. Conforme detalhado na seção anterior, foram utilizados basicamente dois tipos de redes neurais: TDNN (Time Delay Neural Network), formada por Percepton Multicamadas, e uma RNN (Recurrent Neural Network).

A topologia da rede TDNN consiste em 13 entradas obtidas empiricamente, sendo que tal quantidade engloba um período de 12 meses abordando aspectos de sazonalidade e ainda compara o último mês do período anterior com o período presente. A função de ativação dos neurônios da camada escondida é do tipo sigmoidal e a do neurônio de saída é linear.

A partir da verificação e análise dos dados, constatou-se que todos os onze segmentos apresentaram características de séries temporais não-estacionárias, como mostra a Figura 3. A curva em azul é a função de auto-correlação de um sinal de ruído-branco e a curva em vermelho é a função de auto-correlação da série temporal transformada.

Conforme Pindyck e Rubinfeld (1997), funções de auto-correlação de séries temporais estacionárias possuem um coeficiente de correlação que cai rapidamente conforme o deslocamento do tempo, $k$.

A conclusão se fez, a partir de então, da variação gradativa e lenta da função de autocorrelação, diferentemente da curva comparativa aleatória que cai rapidamente de acordo com o tempo. Os picos se repetem em alguns gráficos, mostrando também os aspectos de sazonalidades em períodos de 12 meses. 
58
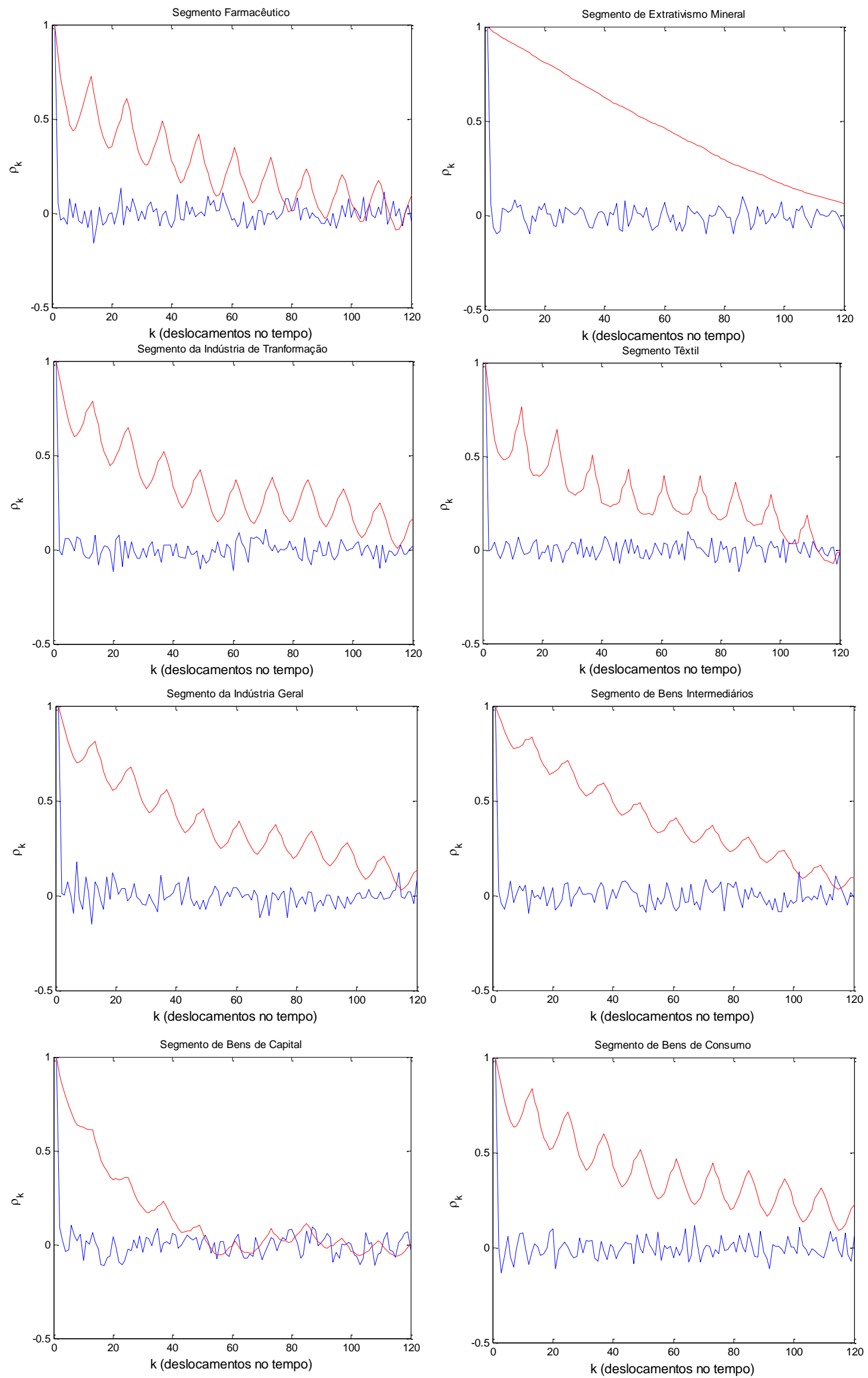

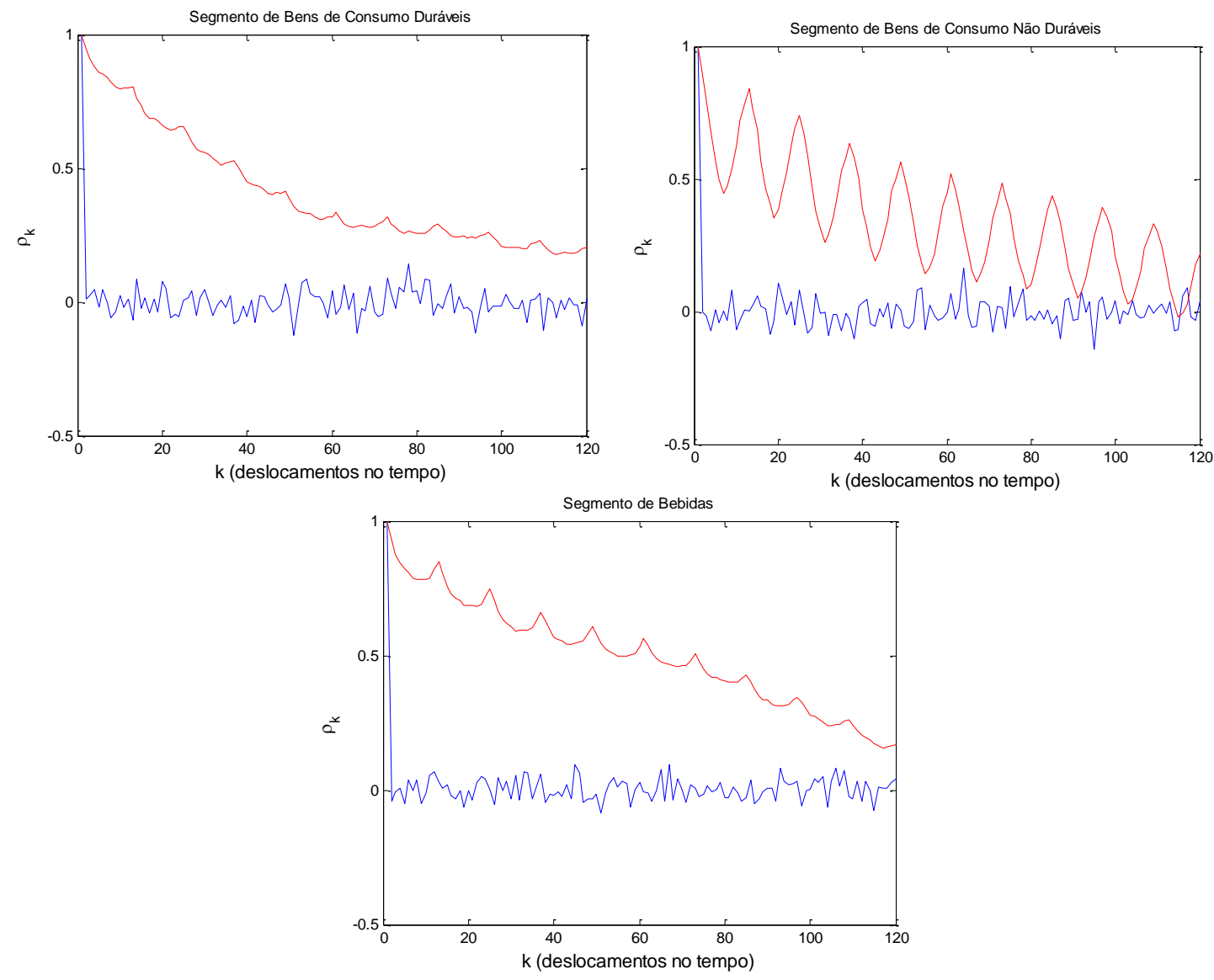

Figura 3 - Função de auto-correlação da série histórica de todos os segmentos.

Embora as TDNN sejam capazes de trabalhar com séries não estacionárias e mesmo com sazonalidades, foi considerado neste trabalho um ajuste dos dados para efeito comparativo dos métodos. Esse ajuste foi feito calculando-se as variações das séries de produção em intervalos de 12 meses, de forma a definir uma nova sequência de dados que terá como resultado uma série de produção praticamente estacionária, conforme mostra a Figura 4.
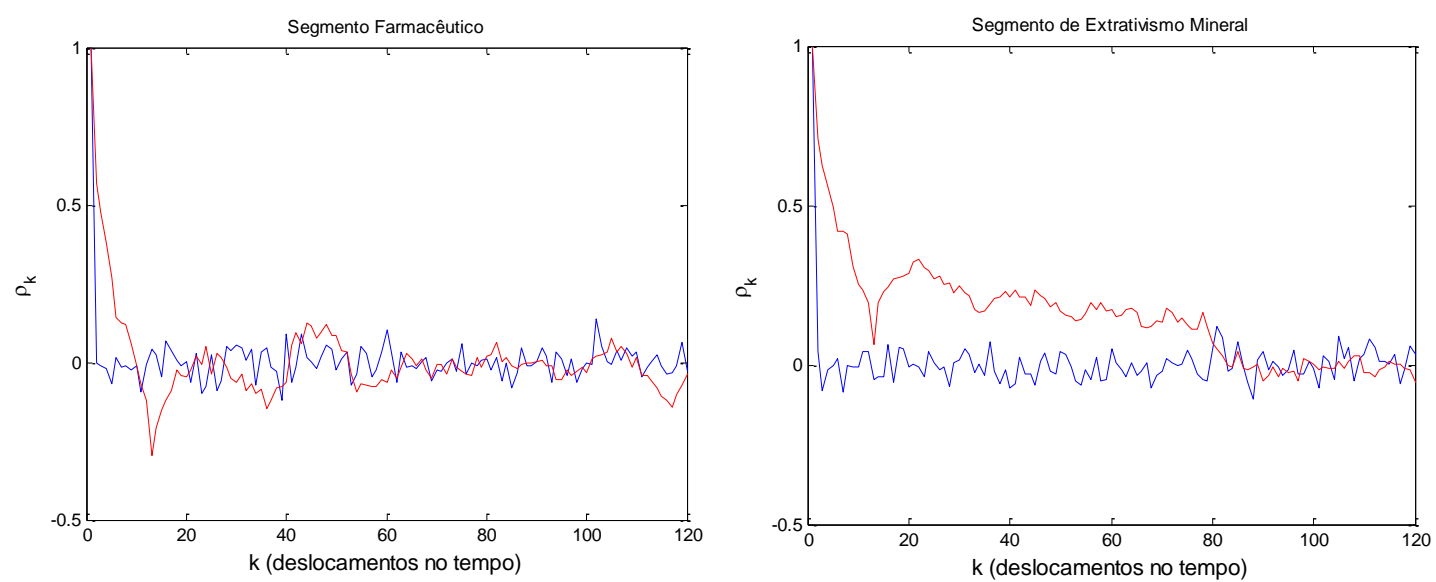
60
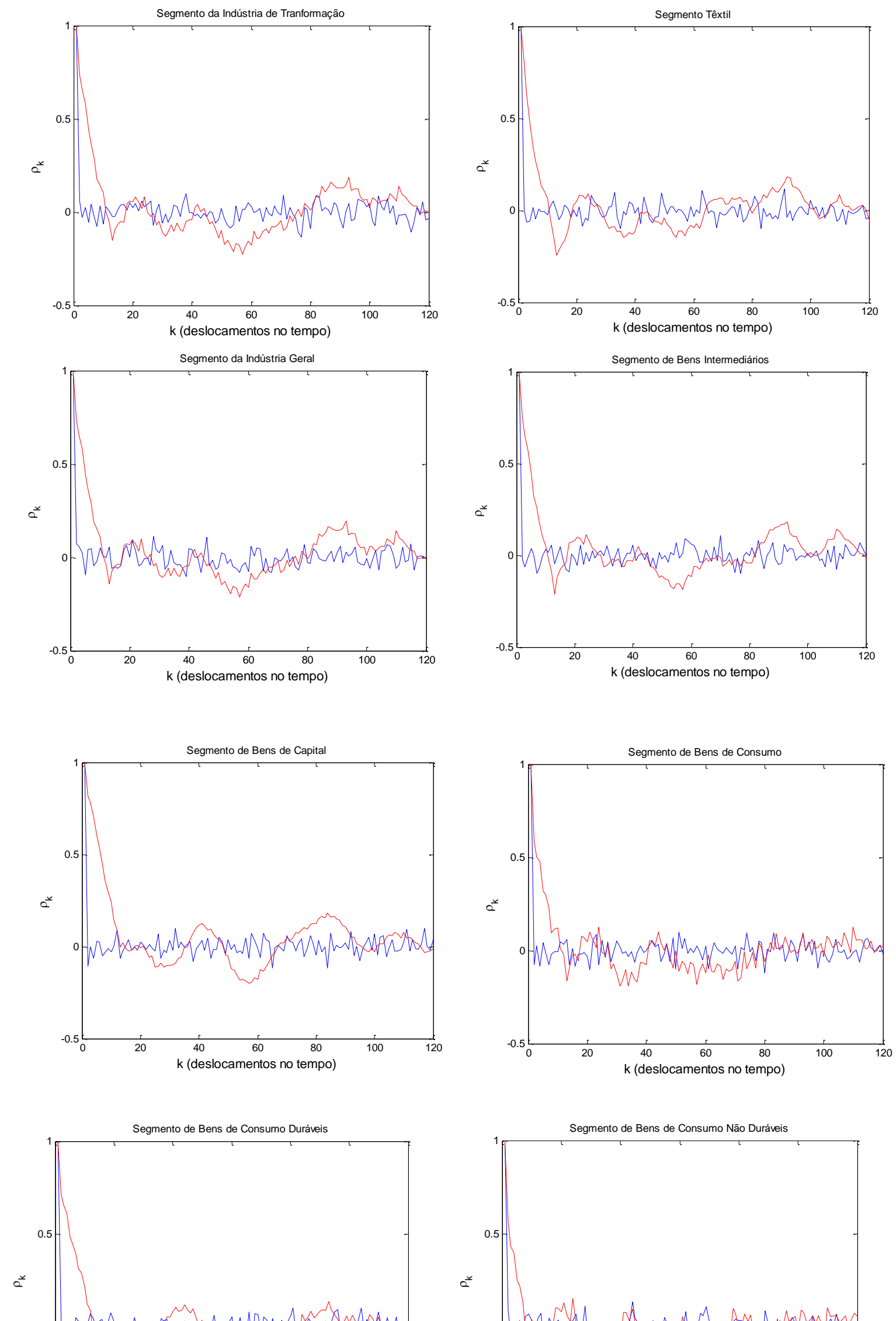


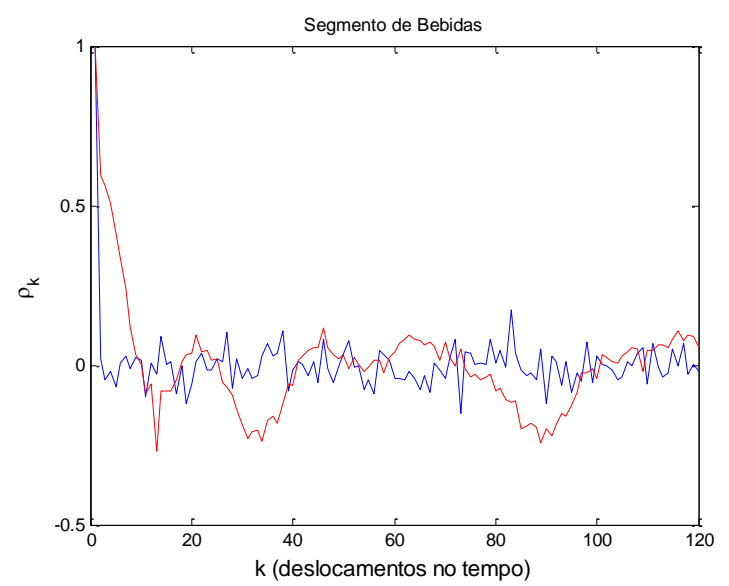

Figura 4 - Função de auto-correlação da sequência temporal das diferenças dos valores em intervalos de 12 meses da série histórica de todos os segmentos.

A partir da análise preliminar utilizou-se inicialmente a rede TDNN diretamente nos dados reais de produção industrial ( $1^{\mathrm{a}}$. Estratégia), e posteriormente foi aplicada a mesma topologia sobre os dados transformados (2a . Estratégia). O tipo de treinamento foi supervisionado, dividido em duas partes: treinamento e simulação, ambos com dados de entrada e saída.

O algoritmo utilizado para o treinamento foi o Levenberg Marquardt, sendo necessárias apenas 25 épocas (passagem completa por todos os dados) de treinamento para se atingir uma boa convergência. $\mathrm{O}$ erro total parte de um valor de aproximadamente 4.000, sendo reduzido a aproximadamente 8 após as 25 passagens, conforme mostra a figura 5 . 


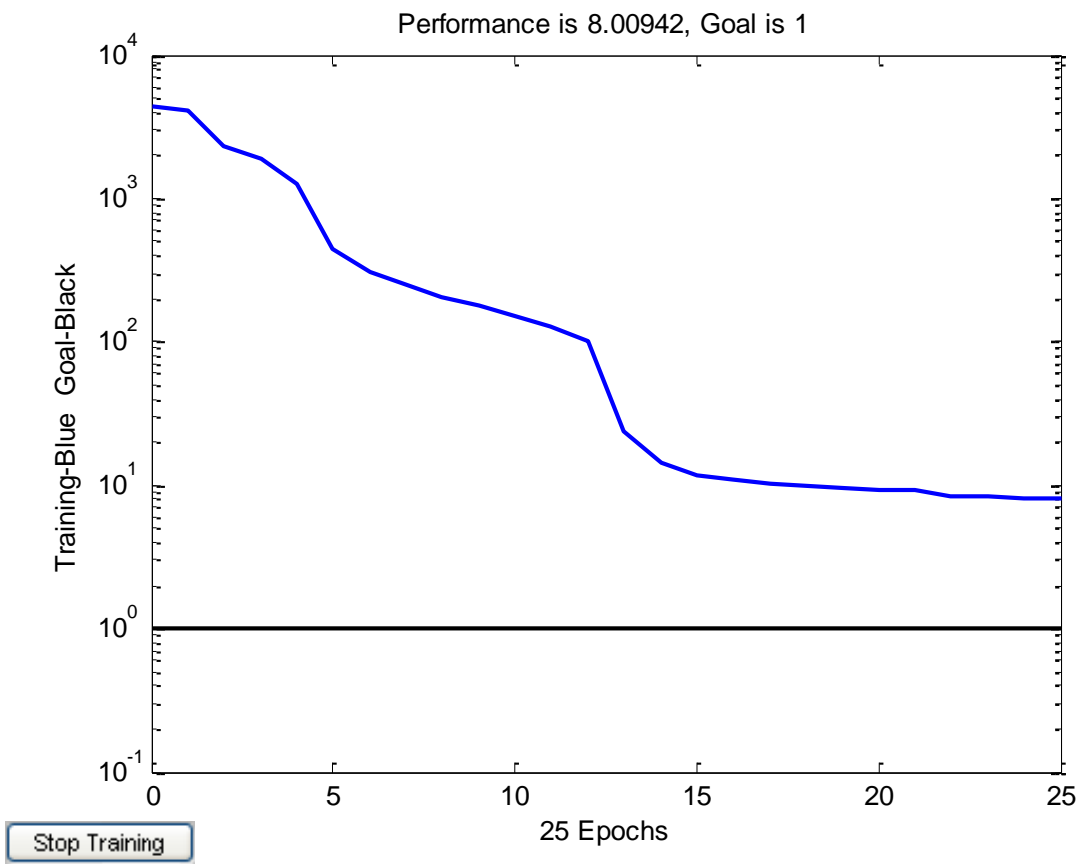

Figura 5 - Curva de aprendizado da RN em 25 épocas.

Para se definir a quantidade de neurônios da camada escondida da rede foram analisados resíduos de simulações realizadas com a quantidade destes neurônios variando de 1 a 25, passando por um teste estatístico de normalidade e calculando a função de auto-correlação; partiu-se da premissa de que os processos são todos estocásticos.

Sendo o segmento farmacêutico aquele que apresentava um conjunto completo de características comuns aos demais segmentos (sazonalidade bastante evidente e um crescimento mais lento), além de oferecer as melhores condições de adaptabilidade do modelo (vide Figura 3), ele foi escolhido para testes de normalidade.

No caso da $1^{\mathrm{a}}$. Estratégia, os dois testes que apresentaram a menor variância na média das observações realizadas foram: a quantidade de 8 neurônios com variância igual a 7,64 e a quantidade de 5 neurônios com variância igual a 7,67. Neste caso, como os valores de variância ficaram muito próximos, foi escolhido aquela com menor número de neurônios na camada escondida, ou seja, 5 neurônios.

$\mathrm{Na} 2^{\text {a }}$. Estratégia, praticamente todos apresentaram padrões de distribuição muito próximos de uma curva normal, embora poucos resíduos tivessem passado no teste de normalidade. A normalidade foi obtida com a quantidade de 3 neurônios, cuja variância ficou em 8,53.

Portanto, a estrutura escolhida da rede na primeira estratégia foi de 13 entradas, em função da própria exigência do modelo em relação ao comportamento sazonal no período de 12 meses, com 5 neurônios na camada escondida e um neurônio na saída, identificada por $\mathrm{N}^{13-5-1 .}$

Como na $2^{\text {a }}$. Estratégia foi eliminado o efeito da sazonalidade, para encontrarmos a quantidade ideal de entradas, foram realizados cinco treinamentos e simulações para cada um dos onze segmentos, variando de 13 a 3; foram calculados também os erros MSE (Mean Square Error) e MAE (Mean Absolute Error).

Para que os resultados das análises dos resíduos de cada segmento apresentassem um comportamento normal, foi necessária a criação de uma variável de penalização relacionada à normalidade dos resíduos e aos erros encontrados, conforme mostra Figura 6. Aquela que apresentou o menor fator de penalização foi a de 4 entradas e, portanto, foi a escolhida para a estrutura da rede neural na $2^{\mathrm{a}}$. Estratégia, identificada por $\mathrm{N}^{4-3-1}$. 


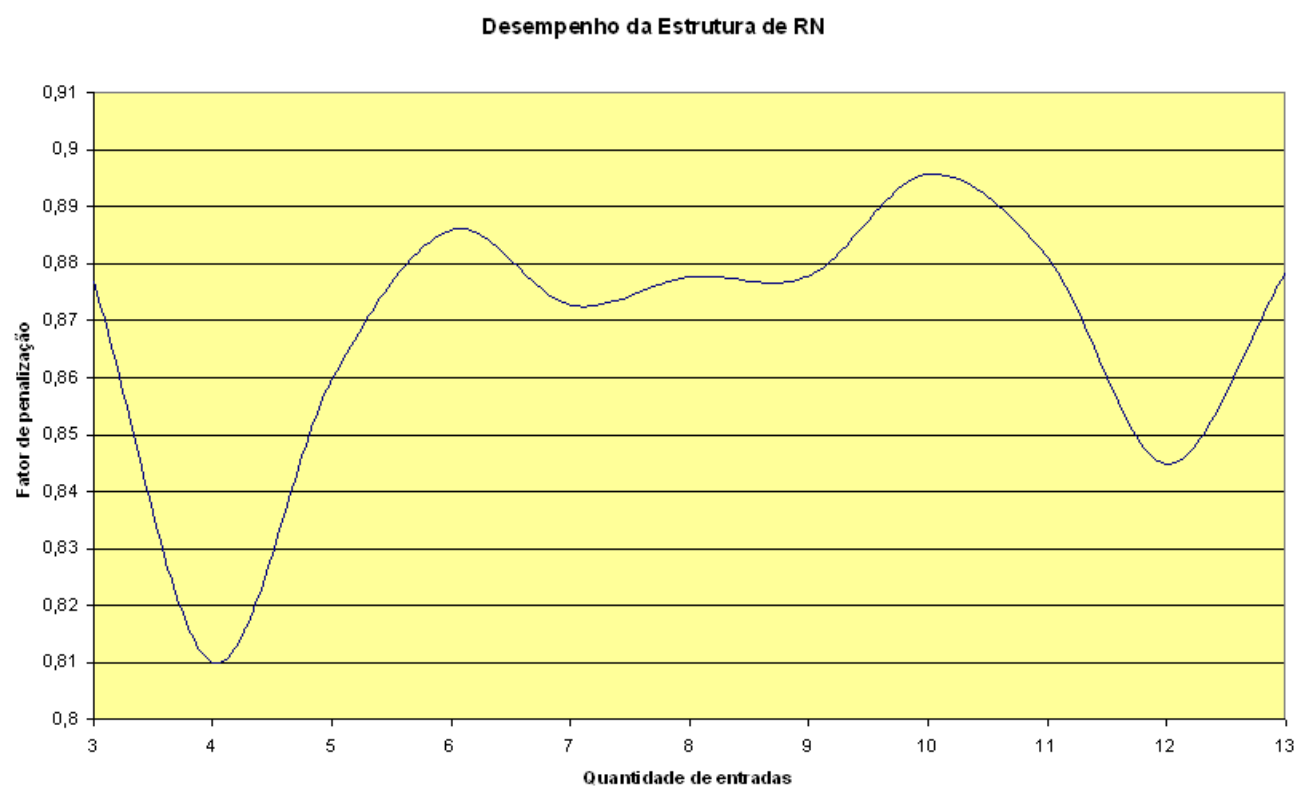

Figura 6 - Resultados da análise das simulações para escolha da quantidade de entradas da RN para aplicação na segunda estratégia.

\section{Análise e Resultados}

Primeiramente, em ambas as estratégias, buscou-se avaliar o segmento de extrativismo mineral, por ele apresentar pouca sazonalidade e crescimento constante, além do segmento farmacêutico por apresentar uma sazonalidade bastante evidente e um crescimento mais lento. Esses dois segmentos nos darão uma primeira idéia da performance de aplicação da rede neural.

Os resultados da primeira estratégia ao segmento de extrativismo mineral encontram-se na figura 7. Verifica-se que a linha azul refere-se aos dados reais e a sobreposição dos dados transformados é representado pela linha rosa; a previsão de demanda entre os meses 395 até 405 é representada pela linha verde.

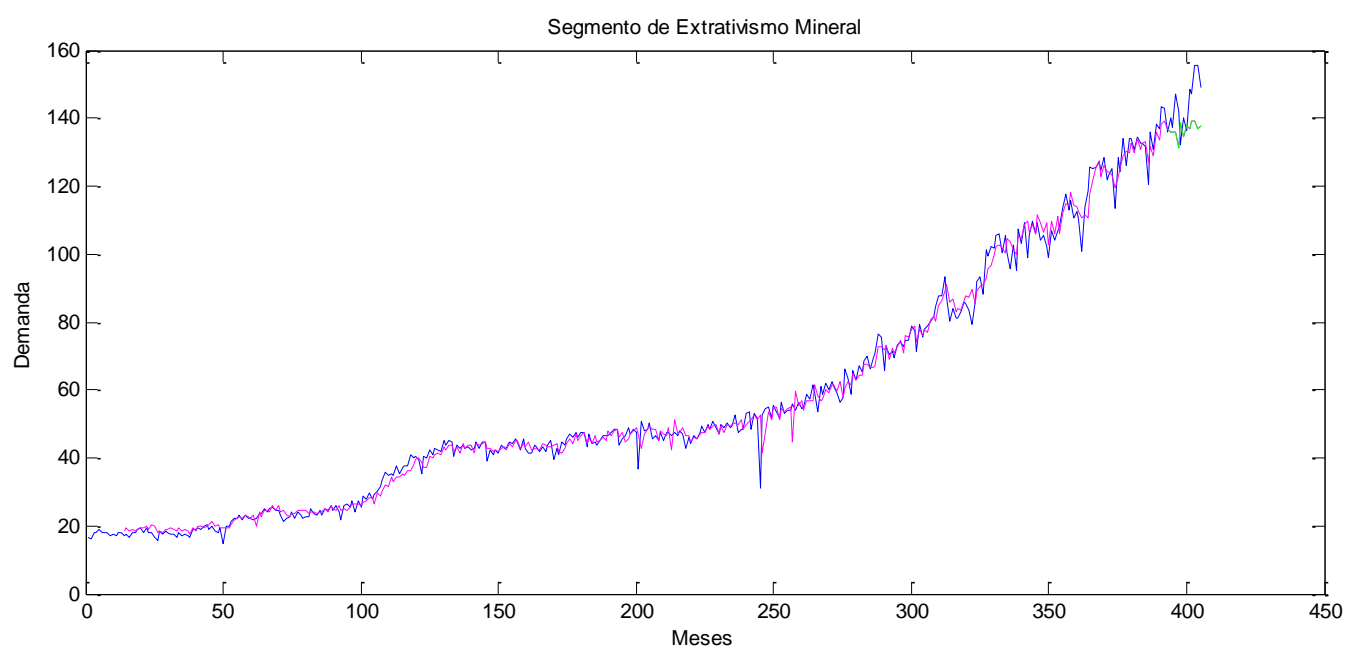


Figura 7 - Resultados das simulações do segmento de extrativismo mineral

Através da análise dos resíduos (vide Figura 8), verifica-se que a variação dos dados reais em relação à previsão chegou próximo aos limites de incerteza, embora o modelo tenha apresentado uma distribuição normal com aceitável excesso de ocorrências próximas da média. As barras verticais mostram intervalos de 1 e 2 desvios padrão.

No caso da primeira previsão, utilizou-se o mesmo $\sigma$ do resíduo, e para as previsões subsequentes, utiliza-se $\sigma \sqrt{l}$ onde 1 é a quantidade de períodos à frente. Este cálculo baseia-se nas estimativas das variâncias $\sigma^{2}$ que se somam e nos resíduos que estão descorrelacionados no tempo (OJA, 1982).
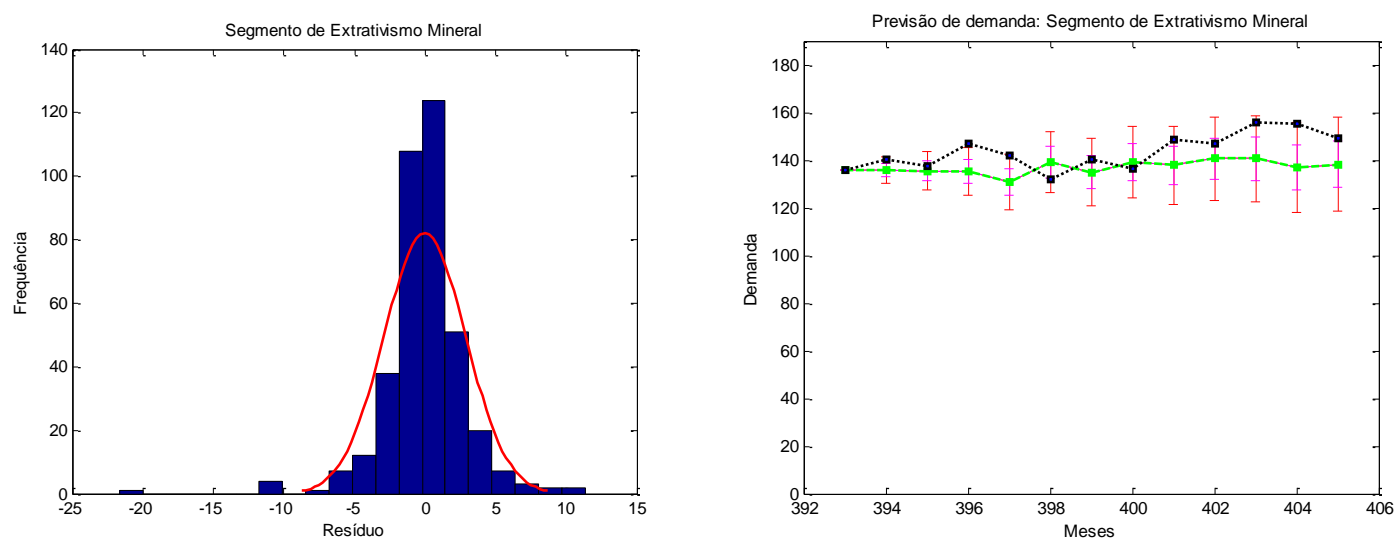

Figura 8 - Distribuição de probabilidade do resíduo com $\mu=-0,0142$ e $\sigma=2,8765$ (Esquerda) e Previsão de demanda do segmento de Extrativismo Mineral utilizando a $1^{\mathrm{a}}$ estratégia de análise. $\mathrm{MSE}=5,8403$ e MAE=16,6178 (Direita).

A Tabela 1 apresenta os pesos utilizados no cálculo da Rede TDNN e que originou a previsão da demanda para o segmento de extrativismo mineral.

Tabela 1 - Pesos e Biases da TDNN $\left(\mathrm{N}^{13-5-1}\right)$ na $1^{\mathrm{a}}$ estratégia do Extrativismo Mineral

\begin{tabular}{|c|c|c|c|c|c|c|c|}
\hline \multicolumn{5}{|c|}{ Pesos das entradas } & \multirow{2}{*}{$\begin{array}{l}\text { Pesos da camada } \\
\text { escondida } \\
w_{j}\end{array}$} & \multicolumn{2}{|l|}{ Biases } \\
\hline$w_{i, 1}$ & $w_{i, 2}$ & $w_{i, 3}$ & $w_{i, 4}$ & $w_{i, 5}$ & & $w_{0, j}$ & $w_{0}$ \\
\hline$-0,0017$ & $-6,9300$ & $-0,4944$ & 18,5079 & 1,7358 & 126,5049 & $-0,6073$ & 28,6583 \\
\hline 0,0039 & $-6,3551$ & 0,6830 & 51,0023 & $-3,5346$ & 0,8638 & $-125,1319$ & \\
\hline$-0,0011$ & 22,0324 & 0,1007 & $-10,2418$ & 2,2065 & 18,0697 & 8,4731 & \\
\hline 0,0006 & $-3,5472$ & $-2,2646$ & 18,6822 & 3,2288 & $-0,1158$ & $-100,9507$ & \\
\hline 0,0000 & $-14,0444$ & 0,2887 & 10,1820 & $-0,5146$ & $-27,1206$ & $-2,9353$ & \\
\hline$-0,0008$ & 29,2165 & $-0,1688$ & 7,2768 & 6,7214 & & & \\
\hline 0,0014 & 3,5420 & $-0,3529$ & 11,8190 & $-18,8722$ & & & \\
\hline$-0,0001$ & $-19,3653$ & 0,5940 & $-42,0550$ & 8,8137 & & & \\
\hline$-0,0009$ & 18,8064 & $-0,3146$ & $-25,7108$ & 1,6403 & & & \\
\hline 0,0008 & $-20,8188$ & 0,8716 & 26,1314 & $-4,5609$ & & & \\
\hline 0,0014 & $-29,6160$ & 1,3460 & $-60,9601$ & 4,8853 & & & \\
\hline 0,0020 & 3,8463 & $-1,0612$ & 13,9632 & $-14,0026$ & & & \\
\hline 0,0031 & 23,9751 & 1,7118 & $-23,1733$ & $-0,8097$ & & & \\
\hline
\end{tabular}


A Figura 9 mostra uma repetição destas análises, porém, considerando agora os dados do segmento Farmacêutico. Percebe-se com mais evidência os ciclos sazonais anuais. Na sequência, a Figura 10 mostra a distribuição de probabilidade e a comparação dos dados reais em relação à previsão, nos mesmos moldes do que foi feito no segmento de Extrativismo Mineral.

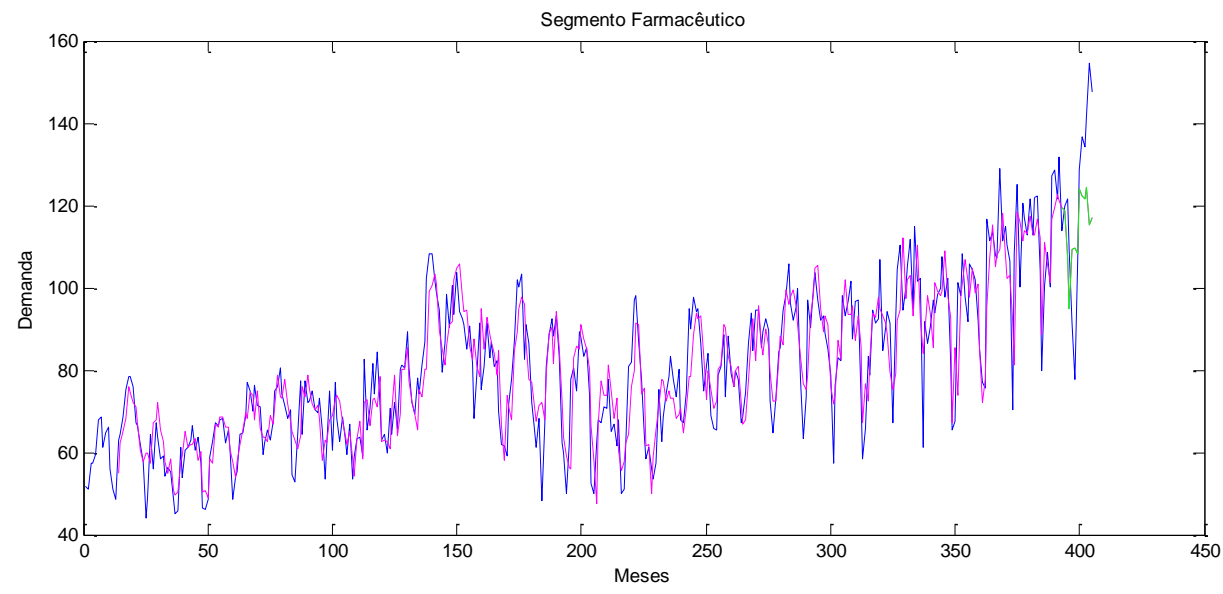

Figura 9 - Resultados das simulações do segmento farmacêutico.
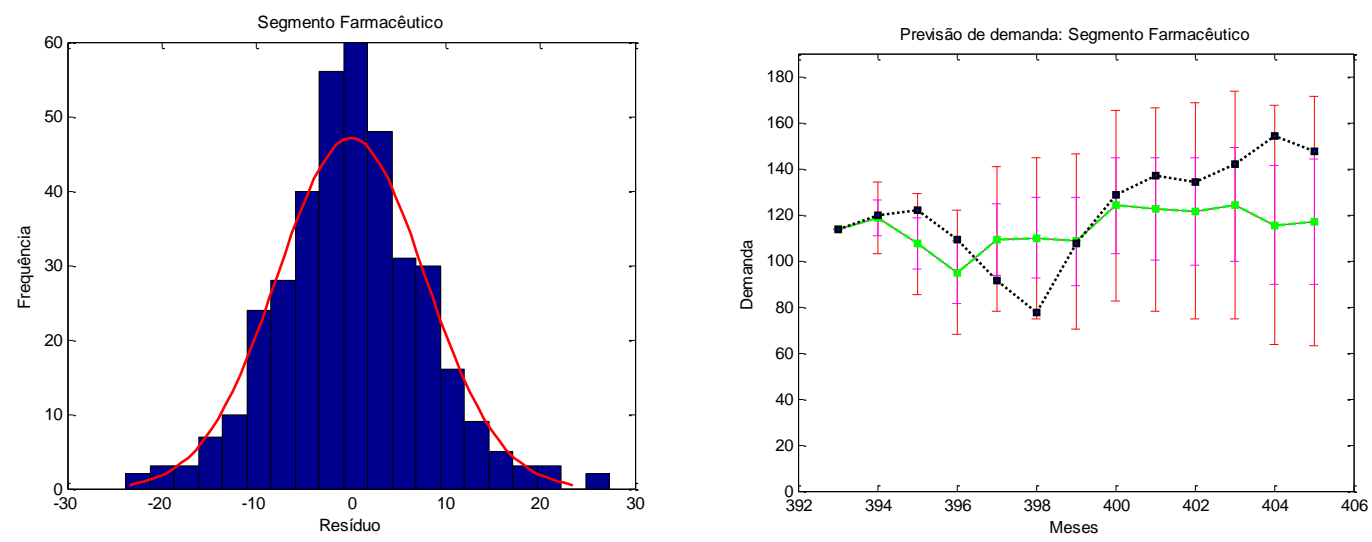

Figura 10 - Distribuição de probabilidade do resíduo com $\mu=0,0031$ e $\sigma=7,8158$ (esquerda) e Previsão de demanda do segmento Farmacêutico utilizando a $1^{\mathrm{a}}$ estratégia de análise. MSE=5,8403 e $\mathrm{MAE}=16,6178$.

Tabela 2 - Pesos e Biases da TDNN $\left(\mathrm{N}^{13-5-1}\right)$ na $1^{\text {a }}$ estratégia do segmento Farmacêutico

\begin{tabular}{|c|c|c|c|c|c|c|c|}
\hline \multicolumn{5}{|c|}{ Pesos das entradas } & \multirow{2}{*}{$\begin{array}{l}\text { Pesos da camada } \\
\text { escondida } \\
w_{j}\end{array}$} & \multicolumn{2}{|l|}{ Biases } \\
\hline$w_{i, 1}$ & $w_{i, 2}$ & $w_{i, 3}$ & $w_{i, 4}$ & $w_{i, 5}$ & & $w_{0, j}$ & $w_{0}$ \\
\hline 97,83995 & $-0,21902$ & $-1,02725$ & $-0,00474$ & 4,89337 & $-0,08996$ & 246,29189 & 85,51668 \\
\hline$-98,78128$ & $-1,99744$ & $-1,44104$ & 0,00750 & $-7,05858$ & $-0,42920$ & 285,89226 & \\
\hline 91,35870 & $-1,56001$ & 5,27727 & 0,00387 & 7,23419 & 0,97392 & $-19,46200$ & \\
\hline$-42,85439$ & 1,31064 & 0,92957 & $-0,00191$ & $-1,88510$ & 62,15531 & $-1,48335$ & \\
\hline 106,12335 & 0,46509 & $-5,49845$ & 0,00208 & 5,34686 & $-2,84345$ & 68,98586 & \\
\hline$-75,41247$ & 0,10952 & 5,70046 & $-0,00247$ & $-12,75571$ & & & \\
\hline$-86,43458$ & $-0,73637$ & $-0,75436$ & 0,00159 & 7,05579 & & & \\
\hline$-90,95014$ & $-0,20782$ & 0,04243 & 0,00063 & $-0,96218$ & & & \\
\hline 32,58869 & 0,76141 & $-1,47557$ & $-0,00155$ & $-1,31476$ & & & \\
\hline 334,34178 & $-1,95373$ & $-4,25216$ & 0,00069 & 2,13308 & & & \\
\hline$-89,57207$ & $-1,88741$ & 3,44475 & $-0,00014$ & $-0,02269$ & & & \\
\hline$-211,33687$ & $-3,08808$ & 1,73796 & 0,00243 & $-0,13788$ & & & \\
\hline 71,38521 & 1,02377 & $-0,98157$ & 0,00888 & $-4,76130$ & & & \\
\hline
\end{tabular}


Como na segunda estratégia, eliminamos os efeitos de sazonalidade através da regra de diferenças de dados de demanda entre intervalos de 12 meses, podendo assim simular os dados em qualquer outro segmento. Analisando-se a Rede Neural com esta nova série de produção aplicada ao segmento de bebidas, verifica-se pela figura 11 uma maior aproximação dos dados transformados (linha tracejada azul) pelo resultado da Rede Neural (linha contínua vermelha).

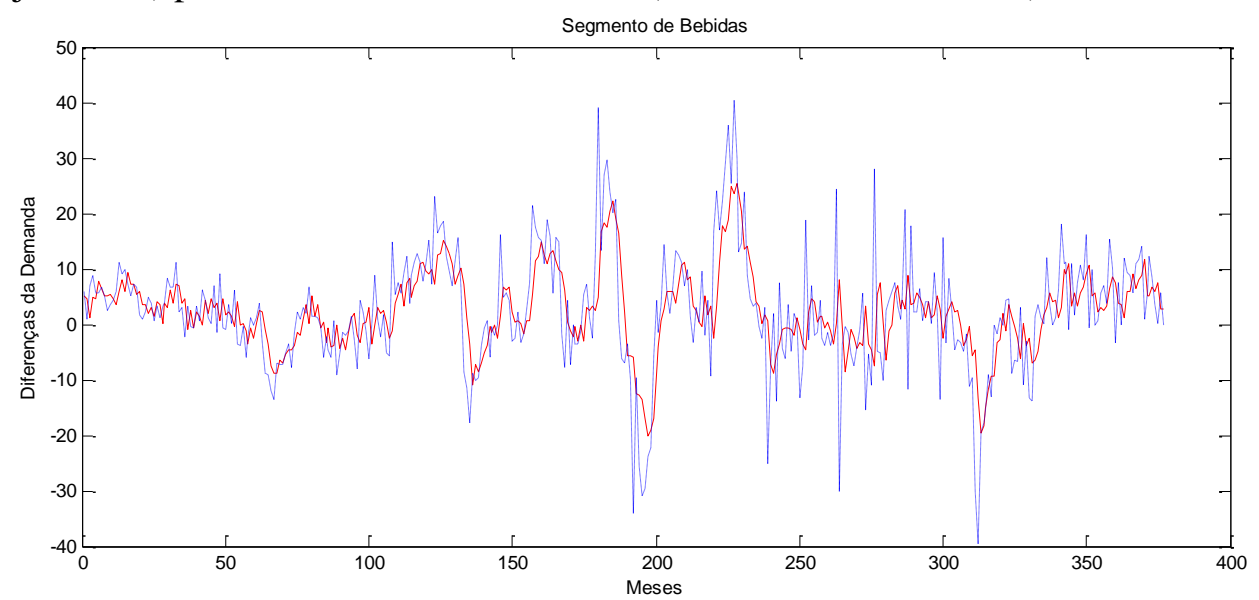

Figura 11 - Dados transformados do segmento de Indústria de Bebidas (azul tracejado) aproximados pela Rede Neural (vermelho contínuo).

Fazendo-se a recomposição do sinal a partir das diferenças estimadas na simulação da Rede Neural, obtivemos os resultados apresentados na Figura 12.

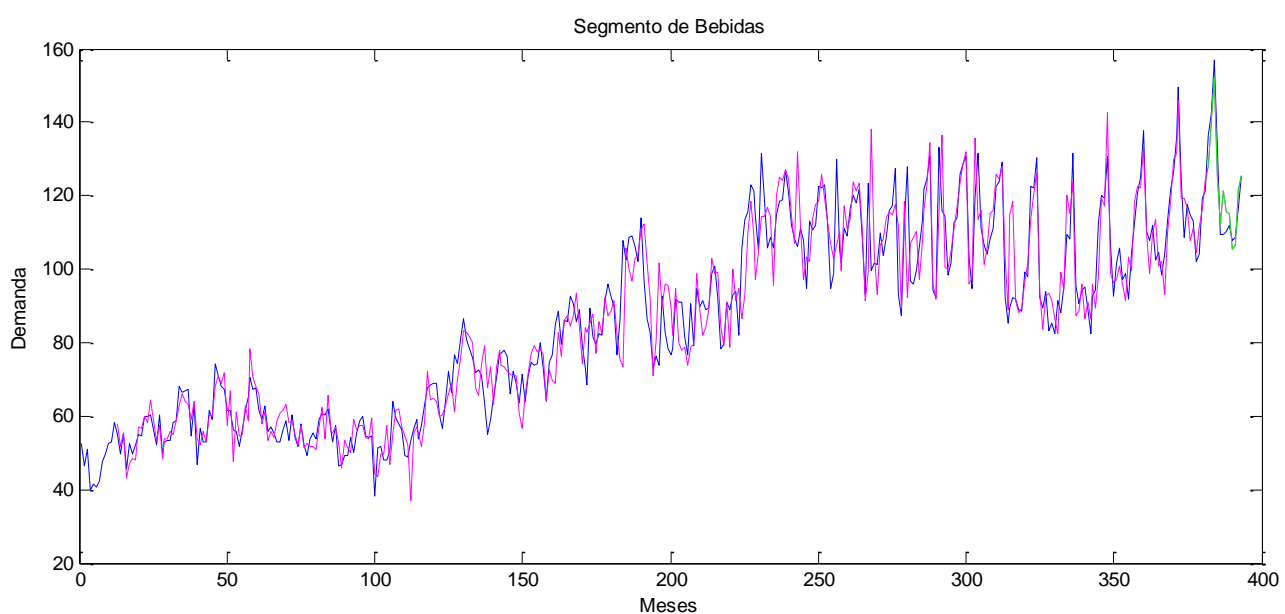

Figura 12 - Resultados das simulações do segmento de Indústria de Bebidas reconstituído a partir da serie de tempo das diferenças. 

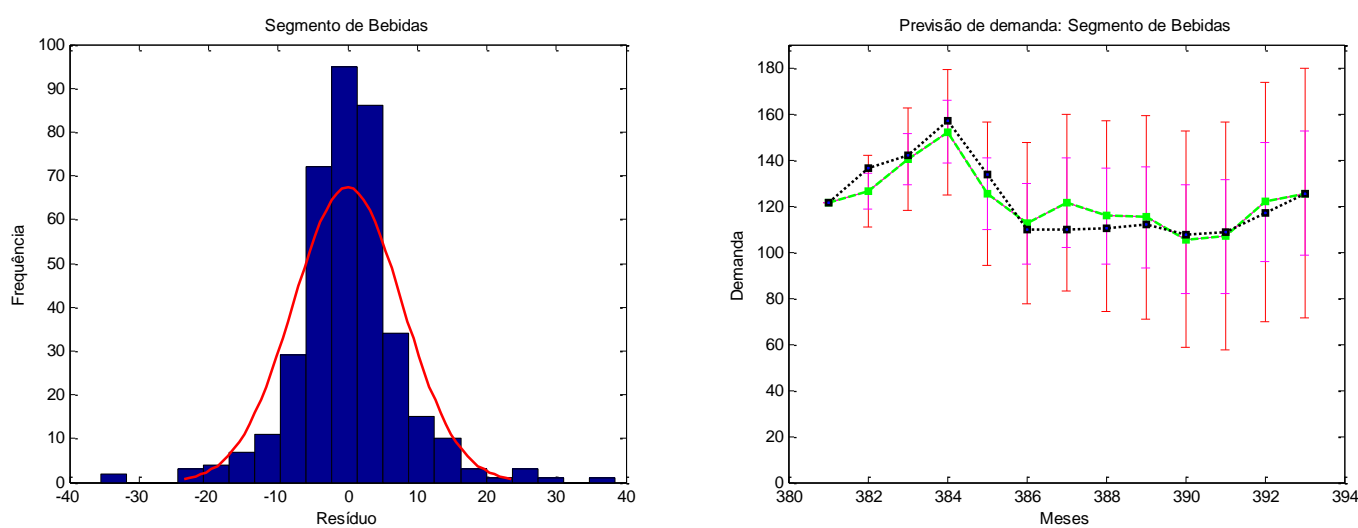

Figura 13 - Distribuição de probabilidade do resíduo com $\mu=0,0158$ e $\sigma=7,8261$ (esquerda) e Previsão de demanda do segmento de Bebidas utilizando a $2^{\mathrm{a}}$ estratégia de análise. MSE=1,7104 e MAE $=4,8085$ (direita).

O teste de normalidade de Kolmogorov-Smirnov foi aplicado para verificar a normalidade dos resíduos. Para um nível de significância de 5\%, concluiu-se que os resíduos seguem uma curva normal, já que a probabilidade associada à estatística foi maior que 0,05 (MONTGOMERY; RUNGER, 2003). Pode-se verificar a partir da figura 13 que a simulação conseguiu acompanhar e descrever o comportamento dos dados reais de forma bastante aproximada. Outra característica observada foi que o resultado da previsão (linha verde) apresentou uma melhor generalização do comportamento sazonal e de crescimento dos dados reais, e que todas as variações ficaram dentro o intervalo de um desvio padrão.

A Tabela 3 mostra os Pesos e Biases da TDNN na $2^{\text {a }}$. Estratégia para o segmento de bebidas.

Tabela 3 - Pesos e Biases da TDNN na $2^{\mathrm{a}}$ estratégia do segmento de bebidas

\begin{tabular}{|c|c|c|c|c|c|}
\hline \multicolumn{3}{|c|}{ Pesos das entradas } & \multirow{2}{*}{$\begin{array}{l}\text { Pesos da camada } \\
\text { escondida } \\
\qquad w_{j}\end{array}$} & \multicolumn{2}{|l|}{ Biases } \\
\hline$w_{i, 1}$ & $w_{i, 2}$ & $w_{i, 3}$ & & $w_{0, j}$ & $w_{0}$ \\
\hline$-0,5738$ & $-0,0001$ & 4,4431 & 1,1910 & 0,1802 & 8,1884 \\
\hline 4,7981 & $-0,0013$ & $-7,5446$ & $-44,9871$ & 0,1768 & \\
\hline$-6,3155$ & $-0,0090$ & 4,6509 & $-0,7493$ & $-2,8697$ & \\
\hline 4,7502 & $-0,0063$ & $-1,2710$ & & & \\
\hline
\end{tabular}

Analogamente aos testes aplicados aos segmentos de Extrativismo Mineral, Farmacêutico e Bebidas, os mesmos foram aplicados aos demais segmentos. Verificou-se que para a $1^{\mathrm{a}}$. Estratégia o comportamento da previsão segue basicamente o mesmo traçado dos dados reais, mas com certa dificuldade em acompanhar o crescimento ou decrescimento dos dados.

$\mathrm{Na}$ aplicação da $1^{\mathrm{a}}$. Estratégia foi constatada que as maiores diferenças foram encontradas no segmento de Bens de Capital.

A maior aproximação dos dados reais com a previsão foi apresentada no segmento de Bens Intermediários e Bens de Consumo Não Duráveis, pelos quais obtivemos os menores erros. 
A aplicação da $2^{\mathrm{a}}$. Estratégia foi bastante eficaz em cada um dos segmentos industriais testados. Igualmente à $1^{\mathrm{a}}$. Estratégia, foram feitas as análises de normalidade e da função de autocorrelação dos dados, cujos resultados apresentaram inexistência de auto-correlação significativa na distribuição apresentada.

Para todos os segmentos, percebe-se que a segunda estratégia foi a mais bem sucedida na previsão de demanda, conforme mostram os valores dos erros MSE e MAE na Tabela 4.

Tabela 4 - Comparação dos erros MSE e MAE entre a TDNN $\left(\mathrm{N}^{13-5-1}\right)$ na $1^{\mathrm{a}}$ estratégia de análise e a $\operatorname{TDNN}\left(\mathrm{N}^{4-3-1}\right)$ na $2^{\mathrm{a}}$ estratégia de análise

\begin{tabular}{|c|c|c|c|c|}
\hline \multirow[t]{2}{*}{ Segmentos de Mercado } & \multicolumn{2}{|c|}{ 1ㄹ Estratégia $\left(\mathrm{N}^{13-5-1}\right)$} & \multicolumn{2}{|c|}{$2^{\text {a }}$ Estratégia $\left(\mathrm{N}^{4-3-1}\right)$} \\
\hline & MSE & MAE & MSE & MAE \\
\hline Farmacêutico & 5,8403 & 16,6178 & 4,6268 & 12,2761 \\
\hline Extrativismo Mineral & 2,8893 & 8,7925 & 1,1954 & 3,9105 \\
\hline Indústria de Transformação & 2,6381 & 7,2603 & 1,5740 & 4,3921 \\
\hline Têxtil & 2,7127 & 6,8294 & 1,4719 & 4,2742 \\
\hline Indústria Geral & 2,5098 & 6,997 & 1,5276 & 4,2792 \\
\hline Bens Intermediários & 1,7803 & 5,1607 & 1,2272 & 3,3421 \\
\hline Bens de Capital & 5,8575 & 17,906 & 4,9333 & 12,9632 \\
\hline Bens de Consumo & 2,8293 & 7,3447 & 1,4335 & 4,0253 \\
\hline Bens de Consumo Duráveis & 5,3399 & 15,2202 & 3,4348 & 9,0343 \\
\hline Bens de Consumo Não-duráveis & 2,3334 & 6,708 & 1,1670 & 3,5839 \\
\hline Bebidas & 4,1866 & 10,5392 & 1,7104 & 4,8085 \\
\hline
\end{tabular}

\section{Conclusões}

Este trabalho aplicou modelos de redes neurais para previsão de demanda de dados de produção de 11 diferentes segmentos do setor industrial.

Os modelos de redes neurais utilizados foram: TDNN (Time Delay Neural Network), formada por Percepton Multicamadas, e RNN (Recurrent Neural Network). A primeira estratégia foi escolhida por ser capaz de trabalhar com séries não-estacionárias, características presentes em todos os segmentos estudados. A segunda estratégia utilizou a mesma topologia de redes neurais, porém, para os dados transformados, de modo a torná-los estacionários, eliminando assim o efeito da sazonalidade. Dessa forma, tanto os dados de entrada como o de saída são diferenças entre dois meses, separados por um intervalo de 12 meses, ou seja, diferenças entre mesmo mês em anos consecutivos.

De uma forma geral, a aplicação do método de Redes Neurais mostrou-se bem aderente ao processo de previsão de demanda, atingindo melhores resultados na $2^{\mathrm{a}}$. Estratégia adotada. Os dados originais foram transformados eliminando-se os efeitos de sazonalidade em intervalos de 12 meses.

A $1^{\text {a }}$. Estratégia, mesmo utilizando uma rede neural mais complexa, foi incapaz de alcançar o desempenho da $2^{\text {a }}$. Estratégia que utilizou uma Rede Neural mais simples a partir da transformação dos dados. A principal razão deste resultado foi a complexidade dos dados originais das séries de produção que são não estacionárias, apresentando média e variância dependentes do tempo e sazonalidades.

Com uma rede mais simples e com séries de dados transformados e praticamente estacionários, a Rede Neural na $2^{\mathrm{a}}$. Estratégia resultou num maior potencial de generalização, proporcionando um aprendizado mais consistente e resultando em pontos que entraram dentro de uma variação máxima de 1 desvio padrão, comprovando a maior habilidade da rede neural em descrever melhores previsões.

Se levarmos em consideração a experiência de quem conduz o processo de previsão e o conhecimento do negócio, e se fizermos uma análise da aplicação de Redes Neurais considerando não somente o resultado da rede em cada segmento, mas também o próprio processo e as etapas de implantação, concluímos como aceitáveis os resultados encontrados.

Portanto, por meio dos resultados e análises dos erros obtidos, pôde-se comprovar e validar a efetiva aplicação da Rede Neural com estrutura Perceptron Multi-Camadas, através de um modelo de 
algoritmo do tipo Levenberg Marquadt, com boa margem de aceitação em quaisquer das duas estratégias propostas.

Como futuras pesquisas, propõe-se um número diferente de neurônios para cada setor em cada estratégia, já que alguns segmentos podem ter menor ou maior complexidade quando comparado com os demais.

\section{Referências}

Aguiar, H.; Caldeira, A. M.; Machado, M. A. S.; SOUZA, R. C.; Tanscheit, R.; Inteligência Computacional Aplicada à Administração, Economia e Engenharia em Matlab. Ed. Thomson Learning, 2007.

Armstrong, J. S. Findings from Evidence Based Forecasting: Methods for Reducing Forecast Error. International Journal of Forecasting, v. 22, p. 583-598, 2006.

Byrne, T.M.M., Moon, M.A., Mentzer, J.T. Motivating the industrial sales force in the sales forecasting process. Industrial Marketing Management, v. 40, n. 1, p. 128-138, 2011.

Braga, A. P., Carvalho, A. P. L.; Ludermir, T. B. 2. ed. Redes Neurais Artificiais: Teoria e Aplicações, LTC, 2007.

Cao, Q.; Ewing, B. T.; Thompson, M.A. Forecasting wind speed with recurrent neural networks. European Journal of Operational Research, v.221, n.1, p.148-154, 2012.

Crone, S.F., Hibon, M., Nikolopoulos, K. Advances in Forecasting with Neural Networks? Empirical Evidence from the NN3 Competition on Time Series Prediction. International Journal of Forecasting, v. 27, n.3, p. 635-960, 2011.

Kaastra, I.; Boyd, M. Designing a Neural Network for Forecasting Financial and Economic Time Series. Neurocomputing, v.10, p.215-236, 1996.

Khashei, M., Hejazi, S. R., Bijari, M. A. A New Hybrid Artificial Neural Networks and Fuzzy Regression Model for Time Series Forecasting. Fuzzy Sets and Systems, v. 159, n.7, p. 769-786, 2008.

Kourentzes, N. Intermittent demand forecasts with neural networks. International Journal of Production Economics, v. 143, n.1, p.198-206, 2013.

Kuo, R. J., Xue, K. C. A Decision Support System for Sales Forecasting through Fuzzy Neural Networks with Asymmetric Fuzzy Weights. Decision Support Systems, v. 24, n. 2, p. 105-126, 2008.

Maccllosh, W. S.; PITTS, W. Alogical calculus of the ideas immanent in nervous activity. Bulletin of Mathematical Biophysics, v.5, p. 115-133, 1943.

Montgomery, D. C.; Runger, G. C. Estatística aplicada e probabilidade para engenheiros. Editora LTC, 2003.

Oja, E. Neural Networks: A Simplified Neuron Model as a Principal Components Analizer. Journal of Math, v. 15, p. 267-273, 1982.

Pindyck, R. S; Rubinfeld, D. L. Econometric Models and Economic Forecasts. 4.ed. McGraw-Hill, ISBN 0-07-050098-3, 1997.

Slack, N., Chambers, S., Johnston, R. Administração de Produção. 2.ed. São Paulo: Atlas, 2009.

Stevenson, W. J. Administração das Operações de Produção. 6.ed. Rio de Janeiro: LTC, 2001.

Werner, L., Ribeiro, J. L. D. Previsão de Demanda: uma aplicação dos modelos Box-Jenkins. Gestão \& Produção, v.10, n.1, p.47-67, 2003. 
Wray, B.; Palmer, A.; Bejou, D. Using Neural Network Analysis to Evaluate Buyer-Seller Relationships. European Journal of Marketing, v. 28, n. 10, p.32-48, 1994. 